\title{
Recepciones de la pedagogía experiencial de Dewey en diversos enfoques metodológicos: el valor añadido del aprendizaje-servicio
}

The reception of John Dewey's in experiential pedagogy in some methodological approaches: The added value of service learning

\author{
Monike Gezuraga Amundarain*1 \\ monike.gezuraga@ehu.eus \\ Ángela García Pérez** \\ angelagarcia@deusto.es \\ *Universidad del País Vasco, España \\ **Universidad de Deusto, España
}

\section{Resumen:}

John Dewey es la personalidad más representativa de la pedagogía norteamericana. Podríamos decir que los aportes hechos en el campo de la Educación han sido innumerables, destacando el "pragmatismo" como uno de los más valiosos. La lectura de su obra supone una fuente inagotable de ideas y propuestas que merecen ser atendidas. El enfoque de la "Pedagogía experiencial" ha propiciado diversas propuestas educativas que vienen desarrollándose en este último siglo en nuestras universidades: el Aprendizaje Basado en Problemas, el Aprendizaje Basado en Retos, etc., lo cual hace notar la actualidad del mismo.

Citamos aquí algunas de las teorías que

\begin{abstract}
:
John Dewey is the most representative personality in American Pedagogy. Whether his contributions to the field are endless, "Pragmatism" stands out as a especially relevant one. The reading of his work is an inexhaustible source of ideas and proposals that deserve to be explored. Dewey's Experiential Pedagogy has left a mark on various educational proposals that have being developed in the last century in our universities: problem-based learning, challenge-based learning, etc. This demonstrates the current validity of Dewey's approach.

The work presented here is a review of the theories that somehow influenced the thinking of Dewey. We explore the projec-
\end{abstract}

1 Dirección para correspondencia (correspondence address):

Monike Gezuraga Amundarain. Universidad del País Vasco. Departamento de Didáctica y Organización Escolar. Facultad de Educación de Bilbao. Barrio Sarriena, s/n. 48940 - Leioa, Bizkaia (España). 
Recepciones de la pedagogía experiencial de Dewey en diversos enfoques metodológicos: el valor añadido del aprendizaje-servicio

monike Gezuraga amundarain y Ángela García Pérez

de algún modo influyeron en el pensamiento de Dewey, para después atender a la propia proyección que este autor y su pensamiento han tenido en Europa y Latinoamérica. Nos acercaremos también a algunas de las propuestas educativas que mencionábamos y que, sin lugar a dudas, se fundamentan en varios de los elementos sustanciales a esa 'Pedagogía experiencial', los principios de continuidad e interacción, o la proyección social de toda experiencia, entre otros. En relación a este último, destacaremos el Aprendizaje-Servicio, como una propuesta de especial relevancia en el desarrollo de los valores y la conducta prosocial.

\section{Palabras clave:}

Dewey; pedagogía experiencial; metodologías; Aprendizaje Basado en Problemas; Aprendizaje Basado en Proyectos; Aprendizaje Basado en Retos; Aprendizaje-Servicio. tion this author and his ideas have had in Europe and Latin America. We also cover some of the previously-mentioned educational proposals which, no doubt, articulate a number of substantial elements of Experiential Pedagogy, more specifically, the principles of continuity and interaction and the social impact of all experience, among others. Regarding this last idea, we will highlight Service-Learning as a proposal of particular importance in the development of values and prosocial behavior.

\section{Key words:}

Dewey; experiential pedagogy; methodologies; Problem Based Learning; Project Based Learning; Challenged Based Learning; Service-Learning.

\section{Résumé:}

John Dewey est la personnalité la plus représentative de la pédagogie nord-américaine. Il est possible de dire que les contributions faites dans le domaine de l'Éducation ont été nombreuses, tout en soulignant le "pragmatisme" comme l'une des plus précieuses. La lecture de son œuvre présente une source inépuisable d'idées et de propositions qui méritent d'être approfondies. L'approche de la "Pédagogie expérientielle" a favorisé diverses propositions éducatives qui ont été développées au cours de ce dernier siècle dans nos universités: I'Apprentissage par Problèmes, l'Apprentissage basé sur des défis, etc., ce qui fait remarquer son actualité.

Nous citons ici quelques-unes des théories qui influencèrent d'une certaine manière la pensée de Dewey, pour après répondre à la propre projection que cet auteur et sa pensée ont eu en Europe et Amérique Latine. Une approche sera faite aussi sur certaines propositions éducatives qui ont été mentionnées et qui sont sans doute fondées sur plusieurs des éléments fondamentaux de cette "Pédagogie Expérientielle", des principes de continuité et d'interaction, ou la projection sociale de toute expérience, entre autres. En ce qui concerne ce dernier élément, nous soulignerons également l'Apprentissage par le Service comme proposition d'intérêt particulière pour le développement des valeurs et de la conduite prosociale.

\section{Mots clés:}

Dewey; pédagogie expérientielle; méthodologies; Apprentissage par Problèmes; Apprentissage par projets; Apprentissage par défis; Apprentissage par le Service.

Fecha de recepción: 13-12-2016

Fecha de aceptación: 06-02-2017 


\section{Introducción}

El tiempo actual requiere de profundos cambios sociales y estos a su vez exigen cambios educativos, es por ello que pretendemos identificar "nuevas" propuestas metodológicas que consideramos vinculadas a la proyección de la pedagogía experiencial de John Dewey. Realizamos este estudio desde un compendio común de rasgos de la "Experiencia Educativa" que hemos decidido resaltar, describiendo dichas componentes mediante extractos directos de sus libros Democracia y Educación (1995/1916) y Experiencia y Educación (2010/1938). Así, la actualidad del enfoque de Dewey se explicita en la conexión con ciertas metodologías/propuestas metodológicas: Aprendizaje basado en problemas (PBL Problem Based Learning) y Aprendizaje Basado en Proyectos (PBL Project-Based Learning). En esta última categoría recogemos dos enfoques metodológicos: Aprendizaje basado en retos (CBL Challenge Based Learning) y Aprendizaje-Servicio (SL Service Learning), destacando especialmente este último, desde su diferencia con otras tipologías de aprendizaje experiencial como el voluntariado; el servicio comunitario institucional; o las prácticas.

No se puede negar la especial incidencia del mundo anglosajón en el iberoamericano, prueba de ello es la propia acogida de John Dewey. En concreto, en el caso del Aprendizaje-Servicio, esto viene acompañado de la relación entre instituciones y, sobre todo, personas que las conforman. Ocurre lo mismo con los centros de investigación al respecto y grupos promotores. La interrelación de CLAYSS (Centro Latinoamericano de Aprendizaje y Servicio Solidario) con NYLC (National Youth Leadership Council, USA) impulsó la creación en 2005 de la Red Iberoamericana de Aprendizaje-Servicio. Así, dedicamos esta mayor profundización en Aprendizaje-Servicio (con respecto a los otros enfoques experienciales) porque partimos de la hipótesis de una mayor adecuación de este enfoque a los fundamentos de "experiencia educativa" de Dewey, especialmente en cuanto a la proyección social-moral de toda experiencia.

\section{Fuentes y recepción de la pedagogía experiencial de John Dewey}

La pedagogía de John Dewey refleja el interés interdisciplinar de este filósofo, pedagogo y psicólogo por diversas fuentes, como la influencia de 
Darwin al respecto de la transformación y el cambio; la filosofía de Hegel o la psicología infantil de Hall. Es muy destacable a su vez la influencia de Pestalozzi (Escuela Activa), Decroly o Montessori, así como del Pragmatismo de Peirce y James. Y, desde ahí, se perciben otras influencias con retrospección a las propias fuentes de sus fuentes, como ocurriera en el caso de Pestalozzi con Rousseau. Para "Rousseau: Ios niños están siempre en movimiento; una vida sedentaria les es perjudicial" (Dewey, 1995/1916, p. 104) y en cierta medida esto pudiera haber influido en la concepción de libertad de Dewey, visible por ejemplo desde ideas como que "la quietud y la sumisión forzadas impiden que los alumnos desvelen sus naturalezas" (2010/1938, p. 102). Esto no le impidió ser crítico con parte de sus planteamientos: "Rousseau (...) estaba profundamente equivocado al suponer que éstos (órganos educativos) ofrecen no sólo las condiciones, sino también los fines de su desarrollo" (2010/1938, p. 103). El mismo Dewey realiza este recorrido crítico a lo largo de toda su obra, como en Democracia y Educación (1995/1916), por ejemplo, al analizar las filosofías de la educación: platónica; individualismo de la "ilustración" del siglo XVIII y el idealismo institucional del siglo XIX.

Sin embargo, la mayor fuerza de sus críticas se concentra en Experiencia y Educación (2010/1938), orientadas éstas hacia el modelo determinista de "Eficiencia Social". Los eficientistas han sido un modelo imperante en EEUU hasta 1970, así como en países que lo "exportaron". En dicho modelo, las personas estarían predestinadas por la medición de una inteligencia que se consideraba casi exclusivamente de "herencia genética". Uno de sus defensores fue el psicólogo Thorndike. Dewey luchó con fuerza contra ese paradigma que implicaba un cierto "adiestramiento" de determinados perfiles en las aulas, que conllevaba al determinismo de una sociedad estratificada.

Por otro lado, y en cuanto a continuadores de su pedagogía experiencial, destacamos la difusión a través de John Holt con su reconocido "aprendemos algo haciéndolo, no hay otra manera" (Holt, 2004/1976, p. 13, traducción propia); el aprendizaje y desarrollo experiencial de David A. Kolb; la semántica de la acción, del filósofo hermenéutico Paul Riocoeur; o su contribución a la tan globalmente extendida Pragmática, con influencias en el desarrollo de diversos modelos como el del Aprendizaje basado en Competencias (Jover y García, 2015). Por supuesto, la contribución de John Dewey y John Holt va mucho más allá del "aprender haciendo", puesto que su aprendizaje mediante la acción tiene una 
fuerte componente liberadora en lo educativo y lo social. De hecho, la estancia de Paulo Freire en Estados Unidos le llevó a estudiar con profundidad la filosofía y pedagogía de Dewey, apropiarlas a las suyas, con ciertas confluencias y distancias, y desde su discurso liberador impulsarlas en su ámbito de influencia iberoamericana (Igelmo, 2013).

Con todo ello, nos atrevemos a recoger los que, como elaboración propia, consideramos rasgos de toda "Experiencia Educativa": principio de continuidad; principio de interacción; procesos de reflexión-acción; actitud de aprender a aprender y las características vinculadas al rol del educador.

\subsection{Experiencia Educativa. Principio de continuidad}

El principio de continuidad concibe la experiencia educativa desde su dimensión de continuum, ya que "aprender por la experiencia es establecer una conexión hacia atrás y hacia adelante entre lo que nosotros hacemos a las cosas y lo que gozamos o sufrimos de las cosas como consecuencia" (Dewey, 1995/1916, p. 125). La "experiencia educativa" precisa de la "ciudanía democrática" y viceversa, podemos observarlo también en la identificación de continuum de la cultura y democracia, como "capacidad para ampliar constantemente el radio de acción y la precisión de la propia percepción de significados" (Dewey, 1995/1916, p. 111).

\subsection{Experiencia Educativa. Principio de interacción}

Las condiciones/factores de la experiencia pueden categorizarse como objetivas (mundo) e internas (yo), sin que ninguna deba "determinar la totalidad del proceso educativo" (Dewey, 2010/1938, p. 84). Así que "una vida comunal auténtica tiene su base en la sociabilidad natural. Pero la vida en comunidad no se organiza de un modo duradero sólo espontáneamente. Requiere pensamiento y planteamiento previos" (Dewey, 2010/1938, p. 97). Una interacción basada en la participación, porque el "ambiente social es educador si se participa" (Dewey, 1995/1916, p. 31).

La existencia o no y el nivel de esta interacción influye también en la disposición de las disciplinas:

la materia en cuestión se aprendió aisladamente; se hallaba como colocada en un compartimento estanco (...) pero como se la segregó y por tanto se la 
desconectó del resto de la experiencia, no es aprovechable para las condiciones actuales de la vida. (Dewey, 2010/1938, p. 89).

Y dentro de dichas materias, la adaptación de cada material:

el principio de interacción demuestra que el fracaso de adaptación del material a las necesidades y capacidades de los individuos puede hacer que una experiencia sea antieducativa tanto como lo hace el fracaso de un individuo para adaptarse al material. (Dewey, 2010/1938, p. 88).

\subsection{Experiencia Educativa. Reflexión-Acción}

Dewey identifica el "método" desde los componentes del pensamiento a través de la acción, nos habla de que el alumno o la alumna tengan una experiencia auténtica:

que surja un problema auténtico dentro de esta situación como un estímulo para el pensamiento; (...) posea la información y haga las observaciones necesarias para tratarlo; (...) que las soluciones sugeridas le hagan ver que él es el responsable de desarrollarlas de un modo ordenado y (...) que tenga la oportunidad de comprobar sus ideas por su aplicación, de aclarar su sentido y de descubrir por sí mismo su validez. (Dewey, 1995/1918, p. 144)

De hecho, "el método científico es el único medio auténtico a nuestra disposición para descubrir la significación de nuestras experiencias diarias en el mundo en el que vivimos" (Dewey, 2010/1936, p. 123). Además, "la reflexión implica también preocupación por el resultado" de la acción (Dewey, 1995/1916, p. 130) y, en el otro sentido, Dewey también alerta del peligro de la acción sin reflexión: "la repetición mecánica para asegurar la eficacia externa del hábito de la destreza motriz sin el acompañamiento del pensamiento señala una deliberada clausura del ambiente para el crecimiento" (1995/1916, p. 52).

\subsection{Experiencia Educativa. Aprender a aprender}

Dewey insta a "adquirir el hábito de aprender a aprender" (1995/1916, p. 49) y a su vez la identifica como "la actitud más importante que se puede formar es la de desear seguir aprendiendo" (2010/1938, p. 90). 
De hecho, la institución educativa debe ser impulsora de ese "deseo de crecimiento continuado" (Dewey, 1995/1916, p. 54).

Este "aprender a aprender" está íntimamente relacionado con la "libertad que es poder, poder para elaborar proyectos, para juzgar acertadamente, para evaluar deseos por las consecuencias que resultarían de su realización; poder para seleccionar y ordenar medios para poner en práctica fines escogidos" (Dewey, 2010/1938, p. 103).

\subsection{Experiencia Educativa. Proyección social}

Dewey se refiere a la proyección social de toda experiencia desde el vínculo a la vida real y desde la concepción de una ciudadanía democrática, basada en un ideal democrático, desde el que compartir valores, oportunidades e intereses mutuos que nos Ileven a una "actividad intencional con propósito, controlada por la percepción de hechos y de sus relaciones recíprocas" (Dewey, 1995/1916, p. 94) y emplear dichos "buenos fines" (p. 93) en un proceso social que transforma (Saltmarsh, 1996).

\subsection{Experiencia Educativa. Rol del educador}

El educador es el responsable del conocimiento de los individuos y del conocimiento de las materias que facilitarán la selección de las actividades que Ileven a una organización social, una organización en la que todos los individuos tendrán la oportunidad de contribuir en algo y en la que las actividades en que todos participen sean el principal sostén de control (Dewey, 2010/1938, p. 97).

El educador como responsable de este conocimiento de las personas, de las materias e, incluso "conocer íntimamente las condiciones de la comunidad local, físicas, históricas, económicas, profesionales, etc. con el fin de utilizarlas como recursos educativos" (Dewey, 2010/1938, p. 24). Todo ello desde una posición de "empática comprensión" (p. 82) hacia sus educandos y hacia la comunidad co-educadora.

A su vez, entender "cada experiencia es una fuerza en movimiento" conlleva como otra "misión del educador ver en qué dirección" va (Dewey, 2010, p. 81), con todas las implicaciones que ello tiene en 
cuanto a inteligencia social y mecanismos para la comunicación. Este contacto entre educador-educandos parte de que "el maestro pierde la posición de amo o dictador exterior y a adopta la de guía de las actividades del grupo" (2010/1938, p. 99).

Finalmente, "el empeño pedagógico de Dewey estuvo dirigido de manera preferente a transformar al profesorado y a la institución educativa" (Beltrán, 2000, p. 57). En relación a ello, Dewey identifica la "Misión de la escuela" (1995/1918, p. 29) desde tres fines que bien pudieran extrapolarse a cualquier entidad educativa: 1) crear espacios de participación en los que dotar de sentido a la acción; 2) no sólo transmitir rasgos de la sociedad, sino mejorarlos; 3) generar oportunidades en ambientes más amplios, con sociedad(es) como suma de muchas.

\title{
3. Enfoques metodológicos actuales al respecto del aprendizaje experiencial. El valor añadido del aprendizaje-servicio
}

\begin{abstract}
Vivimos siempre en el tiempo que vivimos y no en algún otro tiempo, y sólo extrayendo en cada tiempo presente el sentido pleno de cada experiencia presente nos preparamos para hacer la misma cosa en el futuro. Ésta es la única preparación que a la larga cuenta para todo. (Dewey, 2010/1938, p. 90).
\end{abstract}

Dewey formula el proceso de crecimiento siempre en presente, en la acción contextualizada. Así, en la presente sección recogemos diferentes propuestas educativas actuales que tienen una indudable base en la pedagogía experiencial de Dewey. Para realizar este recorrido, en cada caso nos valemos de los rasgos de la "Experiencia Educativa" que hemos identificado y descrito en la sección anterior.

\subsection{Aprendizaje Basado en Problemas}

El Aprendizaje Basado en Problemas (ABProb) es una propuesta que coloca al estudiante en el centro de su propio proceso de aprendizaje, para que profundice adecuadamente en la respuesta al problema que se le ha planteado. Para ello, deberá poner en práctica el análisis de elementos sociológicos, psicológicos y educativos, entre otros (Vizcarro y Juárez, 2008). Este problema podría ser ficticio o real (Arpí et al., 2012). 
Si hablamos de la puesta en práctica del ABProb y si atendemos a diversos desarrollos del modelo, encontraremos diferentes epígrafes en los pasos a dar. De ahí que en algunos casos se hable del 'método de los 7 saltos'; en otros del 'método de los 8 pasos', etc. Nos vamos a centrar en la propuesta de Morales y Landa (2004), que se concreta en las siguientes fases:

- lectura y análisis del escenario del problema,

- Iluvia de ideas de hipótesis sobre aquello que puede estar originando el problema,

- identificación de aquellos elementos sobre los cuales se tiene información previa,

- enumeración de aquello que se cree desconocer: principios, teorías, etc.,

- planificación de la estrategia de estudio-investigación,

- definición del problema,

- búsqueda de información,

- planteamiento de una solución (orientaciones).

Si atendemos a ciertos elementos que han sido y siguen siendo esenciales en el estudio de la concepción de "Experiencia Educativa" de Dewey, diremos que el ABProb representa:

- Un reflejo de lo que son los principios de continuidad y de interacción de la experiencia educativa (Dewey, 2010/1936). El ABProb tiene la capacidad de recoger algo de lo vivenciado, de lo previamente aprendido. Cada estudiante necesita revisar aquello que ya sabe, para orientar el proceso de análisis que será necesario poner en marcha en la solución del problema que se le plantea; de otro lado, el ABProb posibilita que el alumnado entre en interacción con el mundo y las problemáticas que le rodean, posibilita que reflexione acerca de las mismas, de ahí que el contexto de aprendizaje adquiera otra dimensión.

- Una oportunidad para la actividad reflexiva, ya que las y los estudiantes deben reflexionar acerca del contexto en el que se da la problemática, la mejor forma de abordarla, etc. (Allen y White, 2006).

- La presencia de una actitud indagadora, actitud positiva en el aprender a aprender (Atienza, 2008).

- El rol de las y los educadores, quienes acompañan al alumnado 
en la búsqueda de fuentes de información, en la reflexión acerca de las diversas posibilidades de solución al problema, etc. Hablamos de un rol facilitador (Restrepo, s/f).

- Una proyección social (Vega, 2011), pero sólo en el caso de que el proceso se integrase en un contexto real, en una verdadera problemática a la que ofrecer una o más posibles soluciones.

Con todo ello, y si bien el ABProb podría centrarse en una problemática ficticia, entendemos en la medida en la que no se da la necesaria conexión con los agentes implicados, ese aprendizaje pierde fuerza y el alumnado siempre se quedará con la duda de si aquellas posibles "soluciones" que planteó habrían sido efectivas o no. Todo esto nos lleva a reflexionar sobre el "tipo" de experiencia a la cuál exponemos a nuestro alumnado. Otro de los aspectos mencionados es que el ABProb se centra más en los procesos de aprendizaje que en los productos: no se busca obtener un único resultado, se entiende que más de uno puede ser bueno, lo importante es el proceso que ha ocurrido hasta llegar ahí.

Un ejemplo de ABProb sería el caso que la UPV/EHU (2014) presenta a su alumnado de primer curso de Educación Primaria, se trata de un escenario relacionado con las problemáticas de utilización de espacios, y las relaciones de poder que se establecen en un patio escolar. El alumnado trabaja para intentar dar una o más soluciones al mismo, esto les Ilevará, como indicábamos anteriormente, desde la lectura del escenario, el preguntarse acerca de aquello que conocen y desconocen sobre el mismo, pasando por otras fases, hasta la elaboración de las posibles soluciones al problema, y la evaluación de lo aprendido. Se trata por tanto, de un proceso que a modo de ensayo, les ayuda a prepararse de cara a posibles situaciones similares en su futuro profesional.

\subsection{Aprendizaje Basado en Proyectos}

Más allá de la anterior concepción del "problema simulado", encontramos el Aprendizaje Basado en Proyectos (ABProy). Este ABProy orienta al alumnado hacia la búsqueda de una solución concreta, la generación de un producto final, en respuesta a una problemática que se predefine de forma muy concreta. El ABProy proporciona una experiencia de aprendizaje que implica al estudiante en un proyecto complejo y significativo, desarrollando: capacidades, habilidades, actitudes y valores. Esta 
propuesta implica involucrar perfiles diferentes en un equipo de trabajo que se orientará hacia un mismo fin (Maldonado, 2008).

Dentro del ABProy nos encontramos dos tipos de propuestas que entendemos especialmente interesantes dentro de la concepción de lo que es la pedagogía experiencial: una es el Aprendizaje Basado en Retos y otra es el Aprendizaje-Servicio.

\subsubsection{Aprendizaje Basado en Retos}

El Observatorio de Innovación Educativa del Tecnológico (TEC) de Monterrey define el Aprendizaje Basado en Retos (ABR) como "un enfoque pedagógico que involucra activamente al estudiante en una situación problemática real, relevante y de vinculación con el entorno, la cual implica la definición de un reto y la implementación de una solución" (2015, p.3).

En la aplicación del ABR existen diferentes planteamientos de desarrollo. Hemos tomado como referencia la propuesta de Apple (2010), según la cual el proceso se concretaría en las diferentes fases recogidas en la figura 1:
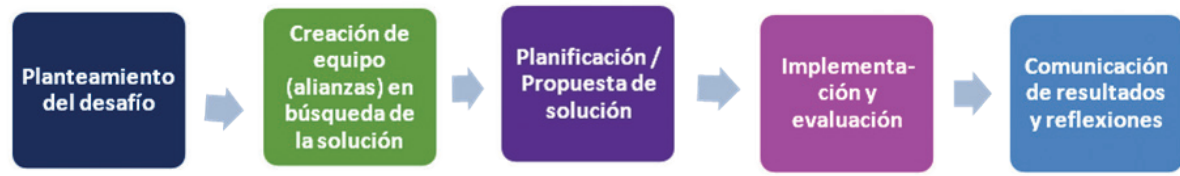

Figura 1. Marco metodológico del ABR (Apple).

Fuente: Adaptado de Apple (2010, pp. 9-15).

Retomando elementos que han sido esenciales en la pedagogía experiencial de Dewey, diremos que el ABR permite:

- Desarrollar los principios de continuidad y de interacción, ya que el reto ante el cual el estudiante se encuentra, le obliga a revisar todos aquellos aprendizajes previos, a indagar y a interactuar con la realidad en la que se busca incidir. El trabajo cooperativo, el diálogo son esenciales en este tipo de procesos (Prieto, 2014).

- Fomentar la reflexión-acción. La reflexión es inherente a estos procesos de indagación donde las y los estudiantes se hacen conscientes del valor de sus aportes, de lo aprendido (Apple, 2010).

- Explicitar la actitud en el aprender a aprender, pues la premisa fundamental es que los estudiantes elijan su desafío (como defienden 
otros movimientos internacionales como "Desing for Change") y, desde ahí, hagan suyo el proyecto en todas sus fases, con especial foco en el diseño. Este empoderamiento del estudiante se refuerza desde el ejercicio de un aprendizaje inductivo como disfrute y descubrimiento permanente.

- Perfilar al educador o educadora como colaboradores en el proceso de aprendizaje (Observatorio de Innovación Educativa del TEC, 2015), no sólo respecto a la relación con su alumnado, sino también a la que establece con otras y otros colegas.

- Alcanzar una proyección social pues, de forma más o menos directa, la mayoría de los proyectos lanzados desde el reto tienen como objetivo la atención de problemáticas sociales (Johnson, Smith, Smythe y Varon, 2009).

Un par de ejemplos sobre Aprendizaje Basado en Retos:

- Chalmers University (Suecia) ha creado el Ilamado Challenge Lab. En este proyecto trabajan profesorado, estudiantes, socios y patrocinadores. Los estudiantes de postgrado están en el centro del proceso, orientando el modo en el que abordar los retos que se les presentan, relacionados con aspectos como la sostenibilidad en el desarrollo arquitectónico-urbano o en la generación de energía (Chalmers, 2014). De hecho, este ABR recuerda mucho al enfoque de "Tiendas de la Ciencia", muy extendido entre los países nórdicos europeos desde 1970 y según el cual algunos doctorandos asumen retos planteados por su comunidad (Pérez Sedeño, 2003).

- A nivel internacional, se extienden iniciativas como los Hackathon. Estos maratones de trabajo (de alrededor de una semana en cada caso) se conciben como encuentro entre programadores informáticos para el desarrollo colaborativo de Software Libre, con un fuerte componente de motivación, trabajo colaborativo con horizontalidad y compromiso con el reto desinteresadamente. Como en otros ABR, los Hackathon pueden llegar a tener conexión con fines sociales concretos (Merpet, Caldeiro y Odetti, 2013) o no. Su emplazamiento en el ámbito local suele ser anual y también se celebran a nivel internacional, como el último en 2015 en Barcelona. 


\subsubsection{Aprendizaje-Servicio}

A pesar de las múltiples definiciones de Aprendizaje-Servicio (A-S) existentes (Jacoby, 2014), pilotamos sobre la concepción que presentan Puig y Palos, siendo A-S una "propuesta educativa que combina procesos de aprendizaje y de servicio a la comunidad en un solo proyecto bien articulado, en el que los participantes se forman al trabajar sobre necesidades reales del entorno con el objetivo de mejorarlo" (2006, p. 60).

Se trata de un proyecto educativo que integra objetivos de aprendizaje y objetivos de servicio, en un proceso de colaboración con la comunidad. Se implementa a través de proyectos solidarios con: una primera fase de motivación hacia el proceso planteado; una segunda de diagnóstico, donde identificar los problemas existentes y priorizar aqueIlos que puede ser más relevante atender; un tercer momento en el que se realiza el diseño, la planificación del proyecto a desarrollar y donde, por último ( $4^{\mathrm{a}}$ fase), se da la ejecución del mismo, tal y como podemos observar en la figura 2:

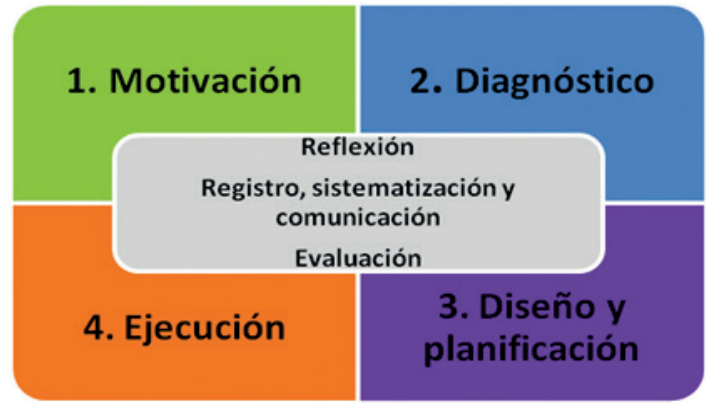

Figura 2. Fases en el desarrollo de un proyecto de A-S. Fuente: Adaptado de Gezuraga (2014, p. 46).

Hay 3 elementos que deben acompañar todo este proceso: la reflexión sobre aquello que estamos haciendo, valorar para qué sirve a otras personas, qué nos permite aprender, etc.; un registro, sistematización y comunicación de todo aquello que se está generando; y una evaluación continua, una revisión del proceso en sí mismo y de los impactos que pueden darse a distintos niveles.

Nuevamente, en relación a la pedagogía experiencial de Dewey, vemos que el A-S posibilita:

- Integrar los principios de continuidad y de interacción. Es necesario proseguir en el anclaje de conocimientos que se ponen e al 
servicio del proyecto. De otro lado, la interacción del grupo de estudiantes con el entorno que le rodea es absolutamente fundamental en este tipo de propuesta, pues esta interacción les permite recoger información de primera mano, redefinir las necesidades que orientaban el proyecto de colaboración en un principio, entender la filosofía que acompaña a una determinada entidad con la que se va a colaborar, etc. (Batlle, 2013).

- Garantizar un proceso de acción reflexionada. Tal y como Páez y Puig señalan, "la actividad reflexiva incrementa la fuerza educativa de cada uno de los dinamismos del Aprendizaje-Servicio y refuerza los vínculos entre dichos dinamismos. Además, la reflexión da sentido social y personal a la implicación en actividades de Aprendizaje-Servicio" (2013, p.15).

- Generar una actitud positiva hacia el aprendizaje. El alumnado inmerso en proyectos de A-S presentan una mayor implicación en sus procesos formativos, más concretamente en la: planificación, desarrollo y evaluación de propuestas (Rubio, 2009).

- Ejercer un rol educador con liderazgo prosocial. En este caso, el/ la docente lejos de ser un espectador, se preocupa por conocer a su alumnado, motiva al grupo, facilita la comunicación en el mismo y conoce en profundidad el proyecto que va a acompañar. Esto implica a su vez un rol activo del acompañamiento educativo en/desde la comunidad, con el educador cuidando las relaciones y tejiendo redes solidarias: como fin para el buen desempeño del proyecto, y como medio de ejemplificación a su alumnado, a quienes implica en la reciprocidad de la acción con los actores comunitarios, junto a los que construyen un significado social compartido (García-Pérez y Mendia, 2015).

- Y como no, vincular a todo ello una proyección social de la experiencia educativa, ya que el A-S no se entiende sin la misma, sin una contribución en la comunidad (Martín, 2009). Así, el A-S hace suya esta convicción de Dewey, ya que este enfoque metodológico tiene un marcado sentido y propósito comunitario de transformación social (Saltmarsh, 1996).

Ahondando en la pedagogía experiencial de Dewey, consideramos necesario profundizar más sobre su recepción en A-S (Giles y Eyler, 1994). Existen varias razones para ello: 
- 'Aprender sirve - Servir enseña': a lo largo de este trabajo venimos hablando de procesos formativos en los que la experiencia es la protagonista y es verdad que, cuando hablamos de ella, podemos hacer referencia a diversas propuestas de aprendizaje experiencial (Furco, 1996): formación a través de unas prácticas de campo (con intencionalidad de aprendizaje); a través del voluntariado (con intencionalidad de servicio solidario); a través de servicio comunitario institucional (con ambas componentes, pero sin una explicitada vinculación a lo curricular), etc. Sin embargo, el A-S tiene la virtud de integrar, de poner en un mismo nivel, el Servicio y el Aprendizaje con el máximo exponente en la intencionalidad de ambos, como se ilustra en la figura 3.

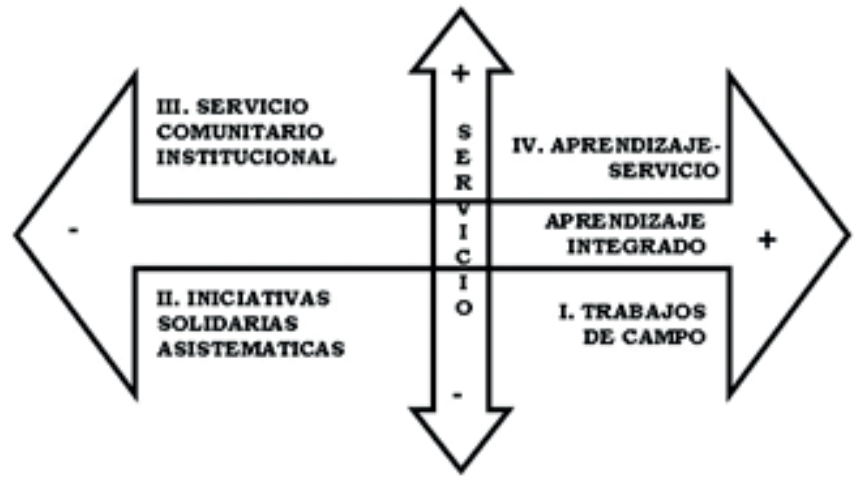

Figura 3. Cuadrantes del aprendizaje experiencial.

Fuente: Tapia (2006, p. 26); adaptado de Service-Learning 2000 Center (1996).

Esta integración genera indudables sinergias: beneficios en el alumnado, el profesorado, la comunidad con la que se colabora y la propia institución educativa (Puig, Batlle, Bosch y Palos, 2007).

- 'El desarrollo de valores' que impulsa el A-S. En el marco de la pedagogía de lo experiencial, Dewey hace referencia a su dimensión moral, identificándola con la dimensión social de dicha experiencia (Dewey, 2010/1938) y aludiendo al "interés constructivo por el bienestar de los demás" que junto a ese desarrollo moral conlleve a un "influjo recíproco" (Dewey, 1995/1916, p. 49). En este sentido, hemos de destacar el potencial que el A-S supone en el desarrollo de valores y conductas prosociales (Roche, 2008).

- 'La integración Docencia-Investigación-Extensión' a través del A-S. 
Esta propuesta ofrece una gran oportunidad a la hora de eliminar las habituales barreras entre la Docencia Universitaria y las que podríamos denominar "hermana mayor" y "hermana menor" de la misma, siendo la Investigación a día de hoy la mayormente valorada en términos de producción científica dentro del mundo académico. El A-S nos demuestra que existe la posibilidad de una integración entre estas funciones (Schlierf, Boni y Lozano, 2010), y no sólo eso, evidencia la posibilidad de un protagonismo estudiantil en las mismas a través de una experiencia de participación directa en el desarrollo de las mismas.

- 'La capacidad de integración a las nuevas modalidades de formación' del A-S. Recientes estudios hacen llamar nuestra atención sobre la rápida adaptación que el A-S está teniendo en relación a las diferentes modalidades de formación on line (Waldner, McGorry y Widener, 2010). El denominado "E Service-Learning" evidencia la posibilidad de una experiencia formativa que va más allá del aquí y ahora, procesos no exentos de dificultades pero que abren una nueva puerta a la participación y experiencia estudiantil.

Destacamos como ejemplo ilustrador de A-S al reconocido "Piletones": estudiantes de la Facultad de Ciencias Veterinarias de la Universidad de Buenos Aires participan en un proyecto que se desarrolla en el contexto de Villa Soldati, entorno en el que convive una población multicultural y carenciada. El alumnado toma parte en actividades relacionadas con: el diagnóstico, la prevención y el control de enfermedades que se transmiten de animales a humanos; y la tenencia responsable de animales y el control poblacional de mascotas. Este proyecto viene implementándose en estrecha colaboración con la Fundación "Margarita Barrientos", entidad que lleva dos décadas trabajando en la villa. El alumnado obtiene un desarrollo potencial de competencias relacionadas con el currículo que cursan (Folgueiras y Martínez Vivot, 2009). Proyectos como este nos inspiran al evidenciar que no sólo puede conseguirse la sostenibilidad de los mismos, sino que además, se parte de una necesidad comunitaria real e INTERDISCIPLINAR. Este proyecto en un principio se concibió como una clínica "únicamente" veterinaria y, en el proceso vivo del mismo, gradualmente se han ido incorporando nuevos "servicios" a la comunidad desde diversas titulaciones: arquitectura, educación, etc. 


\section{Conclusiones}

La revisión construida a lo largo de estas páginas nos ha permitido obtener algunas conclusiones que compartimos a continuación:

- No hay duda de que el pensamiento de Dewey continua despertando interés, teniendo además una amplia proyección en diversos contextos a nivel internacional. Su influencia en diversas propuestas pedagógicas es evidente, en el día a día de nuestras universidades, seguimos buscando las vías mediante las cuales desarrollar una pedagogía experiencial, una pedagogía que ponga a las y los estudiantes en diálogo con la Sociedad. Dewey y su trabajo son una fuente de inspiración a este respecto.

- Podemos decir que los diferentes enfoques metodológicos presentados comparten características similares en cuanto al principio de continuidad, de interacción y a los procesos de reflexión-acción. Sin embargo, se encuentran diferencias en Aprender a Aprender, Proyección Social y Rol del Educador.

En el caso del Aprendizaje Basado en Retos (ABR), se destaca la exigibilidad de que los educandos elijan siempre el desafío. Sin embargo, la proyección social puede ponerse en cuestión dependiendo de cuál sea la finalidad del reto y quiénes sean los socios, pues en algunos casos, se trata de empresas con las que se colabora sin aportar específicamente un "bien común" a la comunidad. En el caso de A-S, este es precisamente un rasgo diferenciador: al menos por definición, el servicio es siempre solidario y debe realizarse en reciprocidad con la comunidad.

El rol del educador presenta una cierta progresión: mientras que en el Aprendizaje Basado en Problemas el docente es guía, en el caso del ABR además es acompañante con una mayor horizontalidad y, finalmente, el A-S suma al papel de facilitador el de líder prosocial, como responsable del cuidado de las relaciones que se den entre todos los participantes, en la construcción de redes solidarias, etc.

- Hemos de subrayar que, a pesar de habernos acercado a propuestas, experiencias educativas que se enmarcan bajo diferentes siglas (ABProb, ABR, A-S...) en algunos elementos es difícil establecer las líneas separatorias entre éstas. ¿El problema no puede ser planteado como un reto?; ¿un reto que implica la colaboración de diferen- 
tes agentes y que se inserta en lo curricular, no puede plantearse como un proyecto de A-S? Entendemos que independientemente de las siglas, lo importante es identificar qué hacemos, cómo lo hacemos, para qué y en alianza con quién. No es poco el profesorado que está convencido de la necesidad de avanzar desde una pedagogía tradicional, hacia una pedagogía más pragmática, hacia procesos de enseñanza-aprendizaje donde encontremos un sentido en aquello que trabajamos (Gezuraga, Egizabal y GarciaPérez, en prensa).

- La interdisciplinariedad de toda experiencia. Si vamos a trabajar sobre problemáticas concretas, retos sociales, nos situamos ante la complejidad de la realidad. Esto nos obliga a adoptar actitudes y formas de hacer distintas como docentes, nos empuja a coordinarnos más y mejor con otras colegas de profesión, con nuestro alumnado y con otros agentes socio comunitarios (Villa, 2013).

- La participación en la Comunidad. Hacemos referencia tanto a la Comunidad educativa, como a la Comunidad-Sociedad, la "Gran Comunidad" de Dewey. El tiempo que nos está tocando vivir requiere de ciudadanas y ciudadanos más comprometidos; esto implica tener capacidad de argumentar, evaluar, tomar decisiones, planificar... en definitiva, participar, y participar sobre temáticas reales, temáticas que nos importan. En ocasiones hemos presenciado procesos en los que se solicitaba la participación de jóvenes, personas adultas, obteniendo un bajo resultado y muchas pueden ser las razones, pero es evidenciable la falta de experiencia o el limitado background al respecto (Palou, Rodriguez y Vilá, 2013). La pedagogía experiencial nos brinda una gran oportunidad en esta línea.

- No podemos dejar de hacer referencia a uno de los aspectos que, a nuestro entender, es de mayor relevancia en todo esto del aprender a través de la experiencia: la contribución en el desarrollo de actitudes y valores prosociales. Nuestras universidades recogen en sus estatutos y planes estratégicos páginas y páginas que hacen referencia a la formación integral de las y los estudiantes, subrayando además elementos como la formación de estudiantes comprometidos, etc. Propuestas como el A-S implican un gran potencial en el desarrollo efectivo de esa formación integral, atendiendo a varias de las cuestiones mencionadas previamente (desarrollo de 
Recepciones de la pedagogía experiencial de Dewey en diversos enfoques metodológicos: el valor añadido del aprendizaje-servicio Monike Gezuraga Amundarain y Ángela García Pérez

competencias unidas a la participación ciudadana), y en la que destacamos con respecto al resto de metodologías/enfoques, la vinculada a la formación en valores a través de la experiencia y, en concreto, a los procesos de reflexión-acción.

La obra de Dewey sigue guiando a gran parte de la comunidad educativa de este globalizado mundo. Una globalización que nos hace pagar no pocos peajes pero que, de otro lado, nos permite seguir compartiendo y aprendiendo de diversos caminos comunes.

\section{Referencias}

Allen, D. y White, H. B. (2006). Estudiantes de pregrado que trabajan como facilitadores de múltiple grupos en el aula. En B. J. Duch, S. E. Groh, D. E. Allen (Eds.), El poder del aprendizaje basado en problemas: una guía práctica para la enseñanza universitaria (pp. 97-112). Lima: Pontificia Universidad Católica de Perú.

Apple. (2011). Challenge based learning: A classroom guide. Recuperado de http://www. apple.com/br/education/docs/CBL_Classroom_Guide_Jan_2011.pdf

Arpí, C., Ávila, P., Baraldés, M., Benito, H., Gutierrez, M.J., Orts, M., ... Rostán, C. (2012). El ABP: origen, modelos y técnicas afines. Aula de Innovación Educativa, 216, 14-18.

Atienza, J. (2008). Aprendizaje Basado en Problemas. En M. J. Labrador Piquer y M. A. Andreu, Metodologías activas. Grupo de Innovación en Metodologías Activas (GIMA) (pp. 11-24). Valencia: Editorial UPV.

Batlle, R. (2013). El Aprendizaje Servicio en España: el contagio de una revolución pedagógica necesaria. Madrid: PPC.

Beltrán, F. (2000). John Dewey. Una Democracia vital. En AA.VV, Pedagogías del siglo XX. Cuadernos de Pedagogía. Especial 25 años. Barcelona: CISSPRAXIS.

Chalmers university. (2014). Challenge Lab. Recuperado de http://media.wix.com/ugd/5 133f3_33c1b235a88046df857483264931289e.pdf

Dewey, J. (1995/1916). Democracia y Educación: una introducción a la filosofía de la educación. Madrid: Morata.

Dewey, J. (2010/1938). Experiencia y Educación. (2a ed.). Madrid: Biblioteca Nueva.

E.U. de Magisterio de Bilbao. (2014). Educación primaria. 1er módulo: FUNCIÓN DOCENTE. GUÍA DEL ALUMNADO. Recuperado de http://www.ehu.eus/documents/2955630/3232350/ATT00010.pdf

Folgueiras, P. y Martínez Vivot, M. (2009). El desarrollo de competencias en la universidad a través del Aprendizaje y Servicio Solidario. RIED, 1(1), 56-76.

Furco, A. (1996). Service-learning: A Balanced Approach to Experiential Education. En Expanding Boundaries: Service and Learning, pp. 2-6. Washington DC: Corporation for National Service.

García-Pérez, A. y Mendia, R. (2015). Acompañamiento educativo: el rol del educador 
Recepciones de la pedagogía experiencial de Dewey en diversos enfoques metodológicos: el valor añadido del aprendizaje-servicio monike Gezuraga amundarain y Ángela García Pérez

en Aprendizaje y Servicio Solidario. Profesorado. Revista de currículum y formación del profesorado, 19(1), 42-58.

Gezuraga, M. (2014). El Aprendizaje-Servicio (A-S) en la Universidad del País Vasco (UPV/EHU): En el camino hacia su institucionalización. (Tesis doctoral inédita). Universidad Nacional de Educación a Distancia.

Gezuraga, M., Egizabal, D. y García-Pérez, A. (en prensa). Catalogación de experiencias de Aprendizaje y Servicio Universitario. En A. Mugarra (Ed.). ApS(U)4: Creando Sinergias (pp. 23-66). Zerbikas Fundazioa.

Giles, D. E., y Eyler, J. (1994). The theoretical roots of service-learning in John Dewey: Toward a theory of service-learning. Michigan Journal of Community Service Learning, $1(1), 77-85$.

Holt, J. (2004/1976). Instead of Education: ways to help people do things better. Holt Associates. Sentient Publications.

Igelmo, J. (2013). Paulo Freire y John Holt: de la educación a la libertad más allá de la educación. Educació i Història: Revista d'Història de l'Educació, 21, 13-35.

Jacoby, B. (2014). Service - Learning essentials. Questions, Answers, and Lessons learned. San Francisco: Jossey-Bass.

Johnson, L. F., Smith, R. S., Smythe, J. T., y Varon, R. K. (2009). Challenge-Based Learning: An Approach for Our Time. Austin, Texas: The New Media Consortium.

Jover, G., y García, A. (2015). Relectura de la educación por competencias desde el pragmatismo de John Dewey. Teoría de la Educación; Educación y Cultura en la Sociedad de la Información, 16(1), 32-43.

Maldonado, M. (2008). Aprendizaje basado en proyectos colaborativos. Una experiencia en educación superior. Laurus, 14(28), 158-180.

Martín, X. (2009). La pedagogía del aprendizaje servicio. En J.M. Puig. (Coord.). (2009). Aprendizaje servicio (ApS). Educación y compromiso cívico (pp. 107-126). Barcelona: GRAÓ.

Merpet, G., Caldeiro, A. y Odetti, O. (2013). Nuestro primer Hackaton. FLACSO Argentina.

Morales, P. y Landa, V. (2004). Aprendizaje Basado en Problemas. Problem - Based Learning. Theoria, 13, 145-157.

Observatorio de Innovación Educativa del Tecnológico de Monterrey. (2015). Reporte EduTrends. Aprendizaje basado en retos. Recuperado de http://www.eduteka.org/pdfdir/edutrends-aprendizaje-basado-en-retos.pdf

Páez, M. y Puig, J.M. (2013). La reflexión en el Aprendizaje-Servicio. RIEJS, 2(2), 13-32.

Palou, B., Rodriguez, M. y Vilá, R. (2013). Convivencia y participación de jóvenes en Sant Boi de Llobregat. Revista de Educación, 361, 252-278.

Pérez Sedeño, E. (2003): Los científicos, el público y la torre de marfil. En Fundación Española para la Ciencia y la Tecnología (FECYT), Percepción Social de la Ciencia y la Tecnología en España, 27, pp. 57-63.

Prieto, A. (2014). Enseñanza inductiva: enseñanza por medio de cuestionamiento y retos. En A. Prieto, D. Díaz y R. Santiago. Metodologías inductivas. El desafío de enseñar mediante el cuestionamiento y los retos. (Capítulo 1). Editor Digital-Text.

Puig, J.M., Batlle, R., Bosch, C. y Palos, J. (2007). Aprendizaje Servicio. Educar para la ciudadanía. Barcelona: Octaedro. 
Recepciones de la pedagogía experiencial de Dewey en diversos enfoques metodológicos: el valor añadido del aprendizaje-servicio Monike Gezuraga Amundarain y Ángela García Pérez

Puig, J.M. y Palos, J. (2006). Rasgos pedagógicos del aprendizaje-servicio. Cuadernos de Pedagogía, 357, 60-63.

Restrepo, B. (s/f). Aprendizaje Basado en Problemas (ABP). Una innovación didáctica para la enseñanza universitaria. Educación y educadores, 8, 9-19.

Roche, R. (2008). Fundamentos psicológicos y pedagógicos del aprendizaje-servicio: la educación para la prosocialidad. Seminarios Internacionales "Aprendizaje y Servicio Solidario" (Antología 1997-2007). Buenos Aires: EUDEBA.

Rubio, L. (2009). El aprendizaje en el aprendizaje servicio. En J.M. Puig. (Coord.). (2009). Aprendizaje servicio (ApS). Educación y compromiso cívico (pp. 91-105). Barcelona: GRAÓ.

Saltmarsh, J. (1996). Education for critical citizenship: John Dewey's contribution to the pedagogy of community service learning. Michigan Journal of Community Service Learning, 3(1), 13-21.

Schlierf, K., Boni, A. y Lozano, J.F. (2010). La transferencia de tecnología participativa desde la universidad: hacia un cambio tecnológico. En M. Martínez (Ed.). Aprendizaje Servicio y Responsabilidad Social de las universidades. (pp. 193-217). Barcelona: Octaedro.

Tapia, M. N. (2006). Aprendizaje y servicio solidario en el sistema educativo y las organizaciones juveniles. Buenos Aires: Ciudad Nueva.

Vega, C.F. (2011). Mirando el Aprendizaje Basado en Problema (ABP) desde las tensiones educativas en su implementación. REDEC, 7(1), 148-166.

Villa, A. (Ed.) (2013). ISUR: Un modelo de evaluación de Innovación Social Universitaria Responsable. Bilbao: Tuning.

Vizcarro, C. y Juárez, E. (2008). ¿Qué es y cómo funciona el Aprendizaje Basado en Problemas? En Julia G. (Coord.). El Aprendizaje Basado en Problemas en la enseñanza universitaria (pp. 17-36). Murcia: Universidad de Murcia.

Waldner, L., McGorry, S. y Widener, M. (2010). Extreme E Service-Learning (XE-SL): a Service-Learning into a $100 \%$ online course, MERLOT Journal of Online Learning and Teaching, 6(4), pp. 839-851. 
